\title{
INSTRUÇÕES SOBRE AS CONSEQUÊNCIAS NEGATIVAS DO COMER E SUA INFLUÊNCIA NO COMPORTAMENTO ALIMENTAR: UM ESTUDO EXPLORATÓRIO.
}

\section{INSTRUCTIONS ON THE NEGATIVE CONSEQUENCES OF EATING AND ITS INFLUENCE ON EATING BEHAVIOR: AN EXPLORATORY STUDY}

\author{
NATÁLIA MARCOURAKIS CALEGARE - ORCID 0000-0003-1048-710X \\ UNIVERSIDADE DE SÃo PAULO, BRASIL \\ DeNigés MAUREL REGIS NeTO - ORCID 0000-0002-5190-7687 \\ PontifíCIA UnIVERSIDADE CATÓLICA, BRASIL \\ LIANE JORGE DE SOUZA DAHÁS - ORCID 0000-0002-3584-1219 \\ UNIVERSIDADE DE SÃo PAULO, BRASIL \\ Centro PARAdigma, Brasil
}

\begin{abstract}
RESUMO
Estudos experimentais investigaram o quanto instruções podem modular a fissura por alimentos. O presente trabalho teve como objetivos averiguar se as instruções exercem controle sobre o consumo de alimento, sobre os relatos de fissura e se há correspondência entre os relatos de fissura e o consumo de alimento. Além disso, teve-se foco em apresentar um método alternativo ao vigente para o estudo de instruções e alimentação, no qual medidas diretas são empregadas para avaliar quanto essas variáveis interagem. Para tal, o experimento contou com três participantes mulheres, no desenho de linha de base múltipla entre participantes. Na linha de base, as participantes foram expostas a imagens dos alimentos de preferência, escalas de fissura e acessavam os alimentos disponíveis para o consumo. Nas sessões experimentais, foram expostas às mesmas imagens, porém eram orientadas a pensar nas consequências negativas de ingerirem os alimentos, considerando sua saúde, peso e aparência corporal; após preencheram as escalas de fissura e tiveram acesso aos alimentos. Foram encontrados indicativos de redução de fissura no decorrer das sessões para os participantes 2 e 3, considerando as análises das médias, o que corrobora a literatura. No entanto, é possível que essa redução se dê em função da repetição da medição da fissura no decorrer das sessões e não necessariamente em função da inserção da variável independente. Não é possível concluir que as instruções exercem controle sobre as variáveis fissura e consumo. Além disso, foram encontrados indicativos de que não há correspondência entre consumo e fissura.
\end{abstract}

Palavras-chave: consumo alimentar, instruções, psicopatologia experimental, fissura.

ABSTRACT
Experimental studies have investigated how much instructions can affect craving for food. This study aimed to verify if the instructions exerted an influence on food consumption, over the craving reports, and whether there is correspondence between craving reports and food consumption. In addition, there was a focus on presenting an alternative method for the study of instructions and eating, in which direct measures were employed to assess how much these variables interacted. The experiment had three female volunteers, and the method design was the multiple baseline among participants. On the baseline, the women were exposed to food images, craving scales, and were given access to food. In the experimental sessions, they were exposed to the same images, but were oriented to think about the negative consequences of ingesting these foods, considering their health, weight and body appearance; after they filled a form with the craving scale, and these foods were made available to them. During the sessions, considering the analyses of the means, participants 2 and 3 manifested indications of craving reduction, which corroborated the literature. However, it is possible that this reduction was due to repetition of the craving measurement during the sessions, and not necessarily the result of insertion of the independent variable. Thus, it is not possible to conclude that the instructions exerted control over the craving and consumption variables. Besides this, indications were found that there was no correspondence between consumption and craving.

Keywords: food consumption; instructions; experimental psychopathology; craving.

Trabalho financiado com Bolsa CAPES concedida à primeira autora e Bolsa do Programa Nacional de Pós-Doutorado (PNPD) e do CNPq - Edital Universal (2014) concedida à terceira autora. E-mail para contato com autores: lianedahas@ gmail.com.

DOI: http://dx.doi.org/10.18542/rebac.v15i1.8325 
Os Transtornos Alimentares (TA) são descritos, pelo DSM V (APA, 2014), como comportamentos alimentares transtornados, que levam a alteração do consumo e/ou absorção dos alimentos, comprometendo a saúde física e psicológica do indivíduo. Alguns dos diagnósticos possíveis de TA são: Anorexia Nervosa (AN), Bulimia Nervosa (BN) e o Transtorno de Compulsão Alimentar (TCA).

De acordo com Udo e Grilo (2018) as taxas de prevalência ao longo da vida para AN, BN e TCA, em uma amostra representativa da população dos Estados Unidos da América, são respectivamente, 0,80\%, 0,28\% e $0,85 \%$. Considerando a prevalência em 12 meses desses mesmos transtornos as taxas são de $0,05 \%$ para $\mathrm{AN}$; $0,14 \% \mathrm{BN}$ e $0,44 \%$ para TCA. A prevalência dos três transtornos é significativamente maior para mulheres do que homens, principalmente considerando AN e BN (Udo \& Grilo, 2018). Segundo meta-análise conduzida por Arcelus, Mitchell, Wales e Nielsen (2011), as taxas de mortalidade são, de maneira geral, altas para todos os diagnósticos de TA, sobretudo para $\mathrm{AN}$, no qual cinco em 1000 pacientes morrem por ano, sendo uma em cinco mortes causada por suicídio, constituindo-se taxas padronizadas de mortalidade superiores às relacionadas aos diagnósticos de esquizofrenia e transtorno bipolar. Dado esse contexto, de altas taxas de mortalidade relacionadas a todos os diagnósticos de TA e tratamentos pouco efetivos, é importante que intervenções com impactos duradouros sejam delineadas. Para tal, é preciso discriminar quais variáveis causam e mantém esses transtornos.

A Psicopatologia Experimental consiste no estudo dos fatores causais e mantenedores de comportamentos relevantes à saúde psicológica e psiquiátrica. Existem duas formas possíveis de testar as relações causais entre variáveis. A primeira forma são experimentos que mimetizam comportamentos considerados "transtornados", porém com força e magnitude brandas e com duração efêmera, em indivíduos saudáveis (Jansen, 2016). A segunda forma consiste na remoção ou redução de um fator em amostras análogas, indivíduos não clínicos que apresentam sintomas subclínicos. Se a variável estiver relacionada de maneira causal, os sintomas serão reduzidos quando a variável for removida (Jansen, 2016).

A produção científica da Psicopatologia Experimental demonstra ser possível estudar experimentalmente comportamentos com relevância clínica, como o comportamento de se alimentar, com o objetivo de explicá-los, prevê-los e alterá-los (Jansen, 2016; Zvolensky, Lejuez, Stuart, \& Curtin, 2001). Alguns estudos experimentais investigaram o quanto estratégias cognitivas podem modular a fissura 1 por alimentos e estimular áreas neurológicas relacionadas ao centro de recompensa ou a centros inibitórios (Hollman et al., 2012; Kober et al., 2010; Siep et al., 2012; Wang et al., 2009; Yokum \& Stice, 2013).

1 Fissura é definida como sendo desejo intenso de consumir alimentos específicos (Pelchat et al., 2004).
Tradicionalmente, a Análise do Comportamento compreende instruções ou regras como estímulos discriminativos descritores de contingências2 (Skinner, 1966/1969, 1974/1976), denominando-se a resposta controlada por tal estímulo de comportamento governado por regra. Neste caso, o indivíduo age como ouvinte e emite a resposta especificada na regra. As estratégias cognitivas, empregadas nos estudos descritos na presente introdução, não passam de descrições de contingências, ou seja, descrições verbais de relações de dependência entre estímulos e respostas. Dessa forma, é possível compreender sob o paradigma analítico-comportamental qual tipo de controle as chamadas "estratégias cognitivas" podem exercer nas variáveis dependentes dos experimentos revisados (Zettle \& Hayes, 1982).

Wang et al. (2009) instruíram os participantes, de ambos os sexos, a inibirem o desejo pela comida apresentada. Os pesquisadores usaram neuroimagens e autorrelatos de fome, desejo por comida e estado de alerta, para medir o controle dessa instrução. Os participantes homens apresentaram supressão da ativação metabólica nas áreas cerebrais relacionadas com a regulação da saciedade e motivação para comer (regiões límbicas e paralímbicas), dado este que não se replicou com participantes mulheres. Considerando os autorrelatos de fome, de estado de alerta e de desejo por comida, homens reportaram diminuição nos três, após a instrução, enquanto mulheres reportaram diminuição apenas nos autorrelatos de fome.

Diferentemente de Wang et al. (2009), Kober et al. (2010) utilizaram instruções em participantes fumantes (homens e mulheres), com o objetivo de modular a fissura de cigarro e de comida. As instruções eram as seguintes: na estratégia now (agora), os participantes deveriam pensar nas consequências imediatas de consumir a substância apresentada nas imagens; na estratégia later (depois) os participantes deveriam pensar nas consequências a longo prazo do consumo dessas substâncias. Foram utilizados neuroimagens e autorrelatos de fissura por comida e por cigarros para medir o quanto as instruções podiam controlar a fissura dos participantes. Pensar nas consequências a longo prazo de consumir as substâncias que apareceram nas imagens (later) demonstrou diminuição no autorrelato de fissura (se comparada com a estratégia now). Considerando as neuroimagens, após o uso da estratégia later, houve aumento na ativação de regiões associadas ao controle cognitivo de maneira geral e com regulação de emoções negativas. Essas ativações foram acompanhadas por uma diminuição na ativação de regiões relacionadas com emoções em geral e com fissura (amígdala).

Semelhante a Kober et al. (2010), Hollmann et al. (2012), instruíram os participantes a (1) admitir o desejo pela comida (condição admit), em algumas sessões, e em outras a (2) pensar nas consequências negativas para vida social e para a saúde de consumir os alimentos

2 Há um debate sobre a definição funcional do comportamento governado por regras, não sendo a proposta skinneriana um consenso na literatura atual (Albuquerque \& Paracampo, 2010). 
apresentados nas imagens (condição regulate). Porém, no experimento mais recente (Kober et al., 2010), todas as participantes eram mulheres e foram utilizadas apenas imagens de comidas como estímulos. Para mensurar o quanto as participantes consideraram difícil empregar as estratégias descritas, foram usados autorrelatos, além de neuroimagens. Por meio dos autorrelatos foi possível observar que as participantes consideraram mais trabalhoso regular o desejo por alimentos palatáveis, do que admitir esse desejo. As neuroimagens demonstraram que a regulação do desejo envolve regiões relacionadas ao controle cognitivo (córtex pré-frontal dorso lateral), o que, segundo Hollmann et al. (2012), sugere que é necessário um alto esforço cognitivo para fortalecer o controle sobre o desejo por alimentos palatáveis, porém não saudáveis. Além dessas regiões cerebrais, foram estimuladas áreas relacionadas a consciência interoceptiva e autorreflexão (ínsula anterior, junção temporo-parietal). Concluiu-se que as participantes foram capazes de regular o desejo por alimento palatáveis.

Em um estudo do mesmo ano, Siep et al. (2012) investigaram respostas neurológicas e autorrelatos de fissura por alimento, dos participantes (mulheres saudáveis) frente a imagens de alimentos altamente palatáveis. As estratégias utilizadas foram: (1) upregulation, na qual as participantes eram instruídas a pensar no cheiro, sabor, textura do alimento; (2) suppresion, em que as participantes eram instruídas a suprimirem os pensamentos sobre a palatabilidade e/ou desejo pelo alimento; (3) cognitive reappraisal, condição na qual as participantes eram instruídas a pensar nas consequências para a saúde de consumir os alimentos mostrados nas imagens e (4) passive viewing, estratégia em que as participantes apenas deveriam olhar para as imagens apresentadas passivamente. A estratégia (1) demonstrou aumento na fissura por alimentos, acessada por questionários e aumentou a ativação de regiões mesocorticais límbicas, relacionadas a recompensa e a motivação apetitiva (Jansen, 2016). A estratégia upregulation demonstrou maior ativação nas regiões mesocorticais límbicas, se comparada às condições (2) e (3). As estratégias (2) e (3) resultaram em fissuras menores do que a estratégia (1), porém não apresentaram diferença entre si. A estratégia suppresion demonstrou maior inibição dessas áreas cerebrais do que cognitive reappraisal.

Estratégias semelhantes foram utilizadas por Yokum e Stice (2013), em um experimento com participantes de ambos os sexos, com Índice de Massa Corpórea (IMC) variado. Os participantes foram orientados a (1) se imaginar comendo o alimento da foto; (2) pensar nas consequências de comer o alimento; (3) pensar nos benefícios de não comer o alimento e (4) suprimir a fissura pelo alimento. Considerando os autorrelatos de fissura pelos alimentos, as estratégias não apresentaram diferença entre si. As instruções (2) e (3) demonstram melhores resultados em aumentar a estimulação em regiões neurológicas inibitórias do que a instrução (4). Apesar disso, a instrução "pensar nos benefícios de não comer" foi levemente mais efetiva na ativação de centros inibitórios (giro frontal superior, córtex pré-frontal ventrolateral), do que a "pensar nas consequências de comer o alimento". Segundo os autores, o IMC não mediou nenhum dos principais efeitos, o que pode se dar devido à pequena variabilidade de peso da amostra de participantes. Apesar de terem sido encontradas diferenças entre gêneros (homens demonstram maior ativação no opérculo frontal do que mulheres, comparando-se a estratégia (4) com a (1) os autores apontam para o fato da amostra ser muito pequena.

Os dados apresentados por Siep et al. (2012) são convergentes com os apresentados por Kober et al. (2010), já que em ambos os estudos a atividade em regiões relacionadas a motivação para comer foram alteradas por meio de instruções (estratégias de controle cognitivo), não só em homens, mas em mulheres também. Porém, diferem dos dados de Wang et al. (2009), nos quais somente em homens foi observada supressão na atividade cerebral de regiões relacionadas a motivação para comer e dos de Yokum e Stice (2012), nos quais a ativação em regiões relacionadas ao controle executivo foi maior em homens do que em mulheres.

Considerando os resultados apresentados por Yokum e Stice (2013) há divergências em relação aos apresentados por Siep et al. (2012) e Kober et al. (2010), tendo em vista os autorrelatos de fissura, já que nos estudos de 2012 e de 2010, os autorrelatos diminuíram após o emprego das estratégias de regulação em comparação com estratégias de ingestão imaginada, enquanto que no experimento de 2013, as estratégias não alteraram os autorrelatos de fissura.

Raynor e Epstein (2003) utilizaram alimentos, bebidas industrializadas e atividades sedentárias para avaliar como a privação e a restrição alimentar influenciam o valor reforçador de alimentos de preferência. Para tal, foi feito um teste de palatabilidade, para garantir que todos os participantes estivessem motivados a ingerir pelo menos um dos alimentos/bebidas disponibilizados. Os participantes eram expostos a uma atividade na qual ganhavam pontos que podiam ser distribuídos para acesso aos alimentos ou acesso à atividade sedentária de preferência. Os autores utilizaram o consumo de alimentos e a pontuação distribuída de cada participante para mensurar o valor reforçador dos alimentos após cada uma dessas condições.

Semelhante ao estudo de Raynor e Epstein (2003), a presente pesquisa utilizou o consumo de alimento como uma das medidas, além de também averiguar o quanto instruções exercem controle sobre os relatos de fissura (Siep et al., 2012). Dessa forma, pretendeu-se produzir um experimento com as características metodológicas descritas por Jansen (2016) como típicas da área da Psicopatologia Experimental. Para tal, foi estudado o quanto o consumo de alimentos altamente palatáveis foi influenciado por instruções.

Os experimentos descritos que apresentam como característica em comum medidas neurofisiológicas e/ou autorrelatos de fissura para correlacionar variáveis não continham nenhuma medida direta do consumo alimentar, como apresentada por Raynor e Epstein (2003). 
Considerando que esse comportamento público (comer) evidencia o quanto situações ambientais, neste caso instruções que funcionam como variáveis independentes (VI), controlam o comportamento alimentar dos participantes, é importante que haja a mensuração de tal variável comportamental (VD) (Baer, Wolf, \& Risley, 1968).

Dessa forma, o presente estudo adicionou uma medida comportamental de consumo de alimentos ao delineamento da pesquisa para então averiguar 1) se há correspondência entre o que os participantes dizem sentir perante os alimentos e o quanto eles efetivamente consomem dos mesmos, 2) se as instruções exercem algum controle sobre o consumo alimentar dos participantes e 3) se as instruções exercem algum controle sobre os relatos de fissura.

\section{MÉTODO}

Considerações acerca dos princípios éticos em pesquisa com humanos. O projeto contendo o método aqui descrito foi enviado e aprovado pelo Comitê de Ética em Pesquisa com Seres Humanos da Universidade Presbiteriana Mackenzie, através da Plataforma Brasil (protocolo CAAE: 74413817.0.0000.0084). Todos os participantes assinaram um Termo de Conhecimento Livre e Esclarecido (também aprovado pelo Comitê de Ética) no qual garantiram estar cientes e de acordo com os termos da pesquisa.

\section{Participantes}

Os participantes foram recrutados por meio da divulgação em redes sociais (e.g. Facebook) e em grupos de Whatsapp de estudantes universitários. Participaram do experimento três mulheres, com idade igual ou maior do que 18 anos, que não tinham pontuado no Eating Attitudes Test -26 (EAT-26) (Garner \& Garfinkel, 1979, traduzido por Bighetti, 2003), não apresentaram distúrbios de tireoide, não estavam em dietas restritivas e não apresentaram IMC considerado abaixo da faixa normal (IMC: 18,5 - 25) (MS, 2014). Os participantes desse experimento foram indivíduos saudáveis, visto que a literatura indica essa população como ideal para participar de experimentos que mimetizem comportamentos considerados transtornados (Jansen, 2016).

\section{Instrumentos}

Eating Attitudes Test - 26 / EAT - 26 (Garner \& Garfinkel, 1979, traduzido por Bighetti, 2003), que avalia a presença de padrões alimentares alterados.

Escala visual analógica para avaliar fome antes das sessões (Figura 1). Os participantes foram orientados a marcar um "X" conforme a proximidade do seu grau de fome.

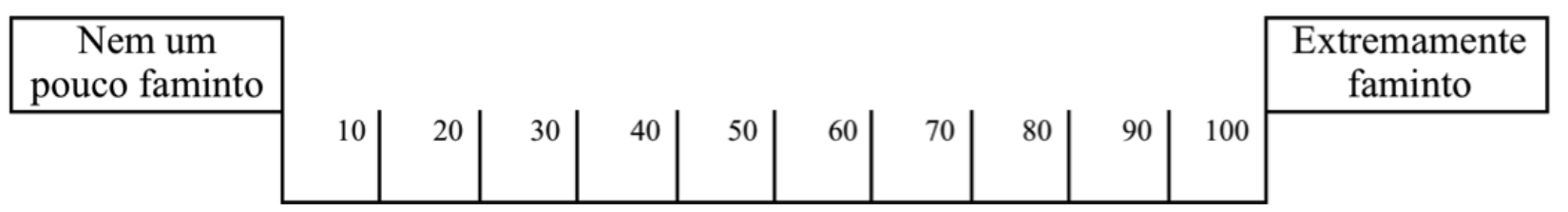

Figura 1. Escala visual analógica de avaliação de fome em milímetros (0 a 100mm).

Escala visual analógica para avaliar fissura pelos alimentos (Figura 2) apresentados nas fotos. A escala de avaliação apresenta uma variação de 0 ("concordo totalmente") a 100 ("discordo totalmente"), que é apresentada com um espaçamento de um milímetro a cada ponto. Os participantes foram orientados a marcarem um "X" conforme a proximidade do seu grau de fissura por um ou mais alimentos. específicos".

"Eu tenho fissura por um ou mais alimentos

\section{Aparatos e Materiais}

As sessões ocorreram em sala disponibilizada pelo Centro Paradigma - Ciências do Comportamento. Foi usada uma apresentação de Power Point em computador, com 15 slides. Cada estímulo (imagem de alimento) apareceu cinco vezes e permaneceu na tela por dez segundos. As imagens eram trocadas automaticamente. Dessa forma foram apresentadas cinco imagens diferentes do mesmo alimento, sendo que cada participante escolheu três alimentos. No total foram apresentadas quinze imagens, disponibilizadas de forma aleatória. Além disso, o experimento contou com uma balança, para pesar os alimentos antes e depois das sessões, e alimentos indicados como preferidos dentro de uma lista oferecida (chocolate - branco, ao leite ou M\&Ms, brigadeiro, bala de goma, bolacha doce recheada, bolo, coxinha, batata Ruffles ou outro tipo de salgadinho, amendoim japonês/ovinho de amendoim, pão de queijo, quibe frito).

\section{Procedimento}

Os participantes receberam um e-mail contendo a lista de alimentos, da qual escolheram seus três preferidos, o Questionário de Pré-Seleção, no qual foram averiguados os critérios de inclusão (idade, peso e altura) e o EAT-26 (Garner \& Garfinkel, 1979, traduzido por Bighetti, 2003), questionário que acessou se o participante pontuava para algum TA. Só foram incluídos no experimento participantes que assinaram o Termo de Consentimento Livre e Esclarecido (TCLE). Os participantes que não preencheram algum critério do Questionário de Pré-Seleção ou que pontuaram mais do no que 21 EAT-26 (Garner \& Garfinkel, 1979, traduzido por Bighetti, 2003) foram excluídos da pesquisa. 


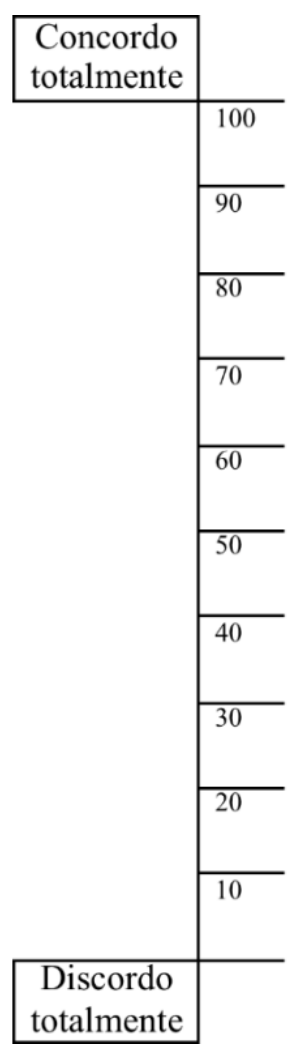

Figura 2. Escala visual analógica de avaliação de fissura em milímetros (0 a 100mm).

Os participantes foram instruídos a virem a todas as fases do experimento estando com, pelo menos, quatro horas de jejum (de alimentos e bebidas, com exceção de água). Era previsto que todos os participantes passassem por quatro sessões experimentais, porém a participante 3 interrompeu sua participação da pesquisa antes de ter completado o número esperado de sessões experimentais.

Para acessar se os participantes estavam, pelo menos, moderadamente privados de alimento, foi aplicada uma escala visual analógica de avaliação da fome, antes do início de todas as fases do experimento.

Os alimentos escolhidos por cada participante foram pesados no início de cada sessão e ao final. A diferença do peso final em relação ao inicial indicou se o consumo se manteve, aumentou ou diminuiu.

O delineamento experimental foi de linha de base múltipla entre sujeitos, ou seja, o momento de inserção da variável independente (VI) variou entre sujeitos, de maneira escalonada, permitindo a comparação entre os comportamentos alimentares (variável dependente) a partir da introdução da variável independente (instrução). O desenho de linha de base múltipla demonstra o efeito da intervenção ao apresentar que a mudança do comportamento só ocorre quando a intervenção é inserida (Kazdin, 1982).
O experimento foi realizado com no mínimo seis e no máximo sete sessões. Foi escolhido de forma aleatória em qual condição cada participante foi alocado.

Condição 1: O participante passou por duas sessões de linha de base e quatro sessões experimentais (total de seis sessões).

Condição 2: O participante passou por três sessões de linha de base e quatro sessões experimentais (total de sete sessões).

Condição 3: O participante passou por quatro sessões de linha de base e duas experimentais (total de seis sessões).

\section{Linha de base}

Os participantes observaram as imagens dos alimentos (apresentadas de forma aleatória), levantados como de preferência e após essa apresentação, preencheram a escala visual analógica de fissura alimentar que indicou fissura por um ou mais dos alimentos apresentados nas imagens. Então, os participantes tiveram acesso a mesa de alimentos (disponibilizados de forma aleatória) e os consumiram. Os alimentos foram pesados antes no início do experimento e no final, para ser possível mensurar o consumo.

\section{Sessão experimental}

Antes de serem expostos às mesmas imagens dos alimentos especificados, os participantes foram orientados a pensarem e escreverem as consequências negativas de ingerirem esses alimentos para a saúde, peso e aparência corporal. Novamente os participantes foram expostos à condição de linha de base na qual foi apresentada uma escala visual analógica que avaliou a fissura por um ou mais alimentos. Em seguida os participantes tiveram acesso a mesa de alimentos.

\section{Procedimento de análise dos resultados}

Os relatos de fome e fissura e o consumo alimentar foram medidos com as escalas analógico visuais de fome e de fissura e a partir do peso consumido, no decorrer das sessões de linha de base e experimentais. Foi observado, através de inspeção visual, se a inserção da instrução (VI) nas sessões experimentais afetou essas três medidas.

\section{RESULTADOS}

Os resultados obtidos na presente pesquisa podem ser vistos na Figura 3, que demonstra o consumo em gramas e a fissura em porcentagem (de zero a cem) por participante, em cada sessão do experimento. A seta preta indica o momento de inserção da variável independente (instrução) para cada participante. 


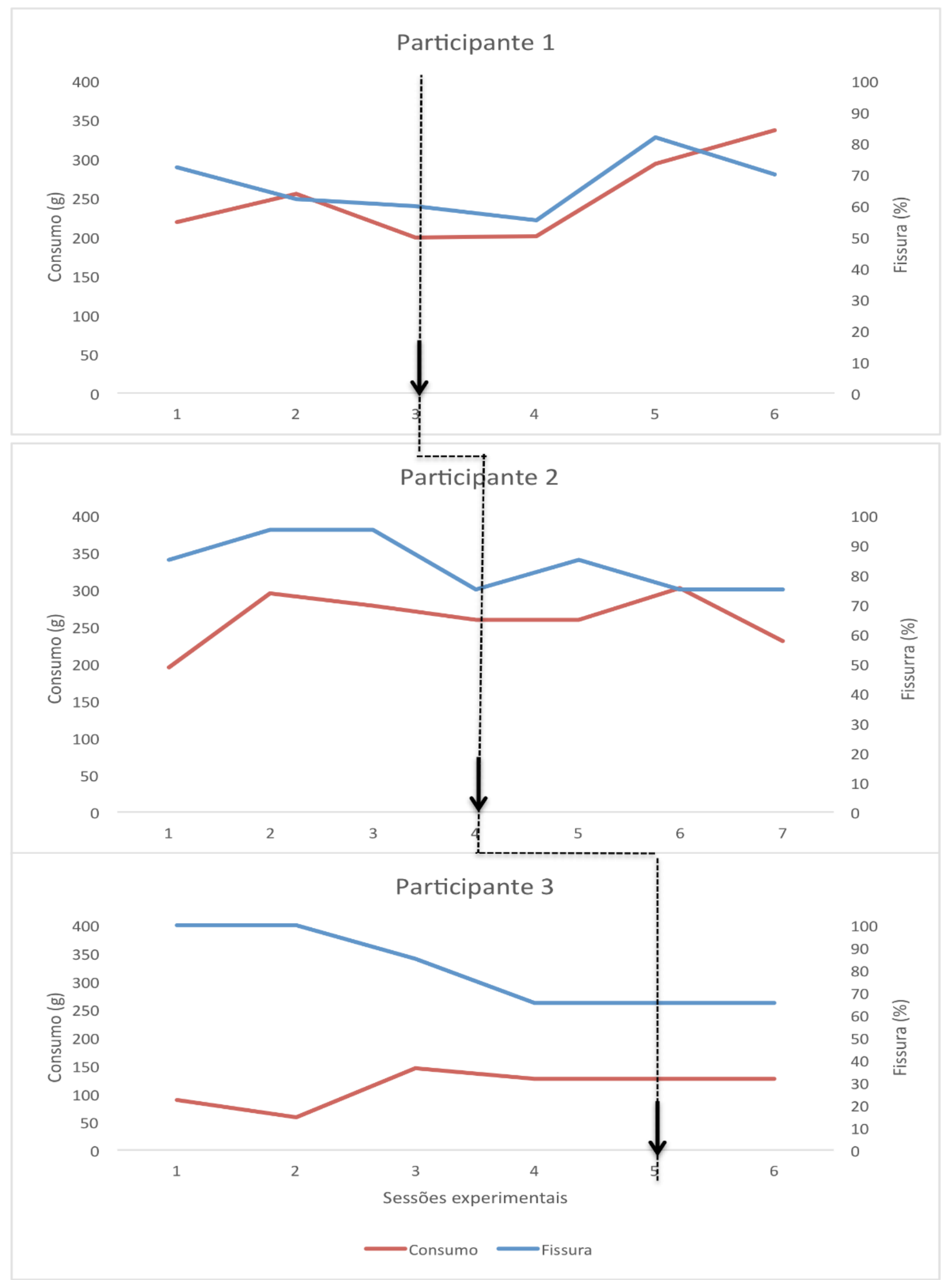

Figura 3. Fissura e consumo por participante no decorrer das sessões. A linha tracejada demarca o início das sessões experimentais realizadas. 
A Participante 1 foi alocada na Condição 1 , na qual passou por duas sessões de linha de base e quatro sessões experimentais. De acordo com a Figura 3, nas duas sessões de linha de base a escala visual analógica demonstrou que o grau de fome da participante foi de $62 \%$ e 78\%, respectivamente. Durante as sessões experimentais o grau de fome foi de $80 \%$ nas duas primeiras sessões, $82 \%$ na terceira e $70 \%$ na quarta. Considerando a fissura pelos alimentos, após a apresentação das imagens de alimentos, foram obtidos os dados de $72 \%$ e $62 \%$ nas duas sessões de linha de base. Ao ser introduzida a variável independente (instrução), o relato de fissura caiu para $60 \%$ e $55 \%$, nas duas primeiras sessões experimentais, subiu para $82 \%$ na terceira sessão experimental e na quarta sessão foi registrado $70 \%$ de fissura. Tendo em vista o consumo de alimento durante as sessões de linha de base, foram consumidas $219 \mathrm{~g}$ e $255 \mathrm{~g}$. Durante as sessões experimentais a quantidade de alimento ingerido caiu para $199 \mathrm{~g}$, na primeira sessão experimental e se manteve estável durante a segunda sessão experimental $(200 \mathrm{~g})$. Porém, na terceira e na quarta sessão experimental houve um aumento do consumo de alimentos que passou para $294 \mathrm{~g}$ e $336 \mathrm{~g}$ respectivamente.

A Participante 2 passou pela Condição 2, na qual foi exposta a três sessões de linha de base e a quatro sessões experimentais. Durante as sessões de linha de base a participante registrou 55\%, 75\% e $65 \%$ de grau de fome, respectivamente. Nas quatro sessões experimentais a participante registrou $85 \%, 95 \%$ e $75 \%$ de grau de fome nas duas últimas sessões. Considerando os relatos de fissura pelos alimentos, nas três sessões de linha de base, a participante registrou $85 \%$ na primeira e $95 \%$ na segunda e terceira sessão. Após a introdução da variável independente, foram obtidos os resultados de $75 \%, 85 \%$ e $75 \%$ nas duas últimas sessões, de grau de fissura pelos alimentos. $\mathrm{O}$ consumo de alimento durante as sessões de linha de base foi de $195 \mathrm{~g}, 295 \mathrm{~g}$ e $277 \mathrm{~g}$, respectivamente. A diferença do consumo entre as duas primeiras sessões de linha de base (195g x 295g) pode ter sido influenciada pela alteração da qualidade do alimento - o alimento foi comprado em dois estabelecimentos diferentes, devido à ausência de disponibilidade - e a própria participante mencionou perceber a diferença de qualidade. Após essa verbalização o alimento voltou a ser adquirido no estabelecimento inicial (mesmo da primeira sessão de linha de base). Considerando o consumo de alimento, após introdução da instrução, a participante consumiu $259 \mathrm{~g}$ na primeira sessão experimental, $258 \mathrm{~g}$ na segunda, $301 \mathrm{~g}$ na terceira e $230 \mathrm{~g}$ na quarta sessão experimental.

Alocada na Condição 3, a participante 3 , passou por quatro sessões de linha de base e duas sessões experimentais. Durante as quatro sessões de linha de base, a participante 3 registrou 55\%, 75\%, 52\% e $38 \%$ de grau de fome. Durante as duas sessões experimentais a participante registrou $52 \%$ e $72 \%$ de grau de fome. Tendo em vista os relatos de fissura, após a apresentação das imagens de alimentos, foi registrado $100 \%$ de fissura por um ou mais alimentos, nas duas primeiras sessões de linha de base, $85 \%$ e $65 \%$ nas duas últimas. Após a instrução ser dada, o grau de fissura pelos alimentos foi de $65 \%$ nas duas sessões experimentais realizadas. $\mathrm{O}$ consumo de alimento durante as quatro sessões de linha de base foi, $88 \mathrm{~g}, 58 \mathrm{~g}$, $145 \mathrm{~g}$ e $127 \mathrm{~g}$, respectivamente. Após a introdução da variável independente, o consumo de alimento se manteve estável (126g e 127g).

\section{Correspondência entre fissura e consumo}

Conforme os dados descritos e contidos na Figura 3, é possível perceber que o grau de fissura registrado pelas participantes não corresponde necessariamente à quantidade de alimento consumida. Considerando os dados da Participante 1 é possível analisar que o grau de fissura atribuído (70\%) à sessão 6 , na qual houve maior consumo de alimento (336g), não corresponde ao maior grau de fissura registrado (84\% na sessão 5) durante as sessões. Contudo as sessões com registro de menor consumo dessa participante $(199 \mathrm{~g}$ e $200 \mathrm{~g}$, sessões experimentais 3 e 4 respectivamente) tiveram como registro de grau de fissura as menores porcentagens $(60 \%$ e $55 \%$, respectivamente). Tendo em vista a Participante 2 , a sessão de menor consumo de alimento (195g, sessão de linha de base 1) registra grau de fissura maior (85\%) do que o atribuído a sessão experimental 6 , de maior consumo de alimento (301g e $75 \%$ ). Para o Participante 3 o maior grau de fissura registrado (100\%) foi atribuído às duas primeiras sessões de linha de base, na qual houve menor consumo registrado ( $88 \mathrm{~g}$ e $58 \mathrm{~g}$ ), enquanto que na sessão com maior consumo de alimento (145g, sessão 3 de linha de base) foi registrado $85 \%$ como grau de fissura. Apesar disso, para essa participante percebe-se que nas três últimas sessões experimentais o consumo de alimento se mantém estável $(127 \mathrm{~g}$ x $126 \mathrm{~g}$ x 127g) e o grau de fissura também (65\% nas três sessões).

\section{Média do consumo e média da fissura}

Conforme as Tabelas 1 e 2 é possível perceber que, para todos os participantes, a média do consumo aumentou após a introdução da variável independente. Porém, se considerarmos a fissura por um ou mais alimentos, é possível perceber que para os participantes 2 e 3 , a média de fissura diminuiu após a introdução da instrução, enquanto que para o Participante 1 a média da fissura permaneceu estável (67\% x 66,75\%).

\section{Tendência do consumo e da fissura}

A análise da média das variáveis dependentes (fissura e consumo) demostra que em média o consumo aumentou para todos os participantes após a inserção da VI e que a média da fissura diminuiu para os participantes 2 e 3 e se manteve estável para o Participante 1.

No entanto, ao se analisar os dados por meio de inspeção visual, uma curva de tendência nos permitiria observar que os padrões individuias não são tão homogêneos e 
provavelmetne não são controlados pela inserçao da VI como a análise de média nos permitiria concluir. A VD consumo, durante as sessões de linha de base do Participante 1, apresenta tendência decrescente, enquanto que durante as sessões experimentais a tendência dessa variável é o aumento.

Tabela 1

Média do consumo - A tabela mostra e média do consumo por participante nas fases de linha de base experimental.

\begin{tabular}{lcc}
\hline & \multicolumn{2}{c}{ Média do consumo } \\
\hline & Linha de base & Sessão experimental \\
\hline Participante 1 & $237 \mathrm{~g}$ & $257,25 \mathrm{~g}$ \\
Participante 2 & $255,67 \mathrm{~g}$ & $262 \mathrm{~g}$ \\
Participante 3 & $104,5 \mathrm{~g}$ & $126,5 \mathrm{~g}$ \\
\hline
\end{tabular}

Tabela 2

Média da fissura - A tabela mostra a média da fissura por participante nas fases de linha de base e experimental.

\begin{tabular}{ccc}
\hline & \multicolumn{2}{c}{ Média da fissura } \\
\hline & Linha de base & Sessão experimental \\
\hline Participante 1 & $67 \%$ & $66,75 \%$ \\
Participante 2 & $91,70 \%$ & $77,50 \%$ \\
Participante 3 & $87,50 \%$ & $65 \%$ \\
\hline
\end{tabular}

Para os participantes 2 e 3 , durante as sessões de linha de base, o consumo apresenta tendência de aumentar, enquanto que após a inserção da VI, a tendência dessa variável, para os dois participantes, é a estabilidade, sendo que para o Participante 3 , com mais sessões de linha de base, tal tendência se dá antes mesmo da inserção da VI. Já a VD fissura apresenta uma tendência decrescente para todos os participantes, durante as sessões de linha de base. Considerando as sessões experimentais, a tendência da fissura para o participante 1 é de aumentar, enquanto que para os participante 2 e 3 a tendência é a estabilidade, para o participante 3 , novamente, essa tendência se aprensenta mesmo antes da VI ser apresentada. É possível, portanto, que o consumo tenda a aumentar e que a fissura tenda a diminuir no decorrer das repetidas sessões experimentais, independente da inserção ou não da variável dita independente.

\section{DISCUSSÃO}

O presente estudo apresenta indicativos de redução do relato de fissura no decorrer do experimento (para os participantes 2 e 3), considerando a análise das médias, o que corrobora a literatura previamente apresentada (Siep et al., 2012 e Kober et al., 2010). No entanto, é possível que essa redução se dê em função da repetição do teste de fissura no decorrer das sessões e não necessariamente em função da inserção da VI. Essa análise se faz possível considerando a análise de tendência dos dados apresentada nos resultados: embora haja redução nos relatos de fissura dos participantes 1 e 2 após a inserção da VI, o Participante 3, que foi submetido a um número maior de sessões de linha de base, apresenta essa redução antes mesmo da inserção da VI. Nesse caso, uma análise dos dados considerando apenas as médias das sessões de linha de base e das sessões experimentais poderia favorecer conclusões errôneas. Dessa forma, não é possível concluir que a VI (instrução) exerce de fato controle sobre as variáveis dependentes (fissura e consumo).

Todos os artigos concluíram que foi possível modular a fissura dos participantes perante os alimentos, utilizando instruções que supostamente aumentem o valor aversivo dos alimentos (consequência a longo prazo para a saúde, peso ou supressão do desejo pela comida). Somente o experimento de Yokum e Stice (2013) demonstra que as instruções desse tipo não produziram mudanças nos relatos de fissura. Apenas dois, dos cinco artigos revisados, delinearam uma condição na qual os participantes teriam a estimulação dos alimentos (imagens ou cheiro/gosto do alimento) sem nenhuma instrução relacionada a como os participantes deveriam reagir frente a eles (linha de base) (Wang et al., 2009; Siep et al., 2012). Contudo, um deles não fez medição de fissura durante essa condição (Siep et al., 2012). Os outros três experimentos compararam instruções diferentes entre si, porém não com uma condição livre de instruções (linha de base). Além disso, apenas um experimento fez medidas de fissura em dias diferentes (Wang et al., 2009), tendo todos os outros experimentos feito essa medição diversas vezes no mesmo dia, o que não nos permite comparar nossos dados de tendência com os da literatura.

Apenas Yokum e Stice (2013) concluíram que o uso de instruções foi efetivo para modulação da fissura sem de fato ter apresentado evidências de que houve mudanças nos relatos de fissura após a apresentação das instruções. Os demais autores utilizaram correlatos neurais e os relatos de fissura como medidas para evidenciar a modulação da fissura pelos alimentos, após o emprego de instruções que aumentam o valor aversivo de alimentos altamente palatáveis. Todos eles inferiram que, como as instruções ativaram centros inibitórios ou estimularam menos centros de recompensa, o consumo dos participantes tenderia a ser menor frente a instruções semelhantes à usada nessa pesquisa.

Embora a literatura apresentada (com exceção de Raynor \& Epstein, 2003) não utilize a conceituação de autocontrole de Rachlin (2000), é possível discutir 
seus dados sob a luz desse paradigma: o autor propõe que ao se estudar o fenômeno do autocontrole, se utilize sempre o locus temporal como variável controladora de comportamentos de escolha entre dois (ou mais) esquemas concorrentes; enquanto um esquema apresenta, de maneira atrasada, uma consequência reforçadora de alta magnitude, o outro a apresenta imediatamente, porém com uma magnitude mais baixa. Dessa forma, o comportamento autocontrolado seria aquele que opta pelo esquema com consequência maior, apesar do atraso de sua entrega.

O modelo de autocontrole de Rachlin (2000) permite que comportamentos humanos tidos como impulsivos (sob controle de consequências de menor magnitude e de curto prazo) sejam observados, preditos e controlados a partir da manipulação das variáveis (1) tempo de atraso entre a resposta e a liberação do reforço e (2) magnitude do reforço nos mais diferentes esquemas concorrentes. O comportamento alimentar engloba (assim com o uso de drogas - McKim \& Hancock, 2013) a fissura - "craving", a busca - "seeking" e a autoadministração "self-administration", e é, portanto, passível de ter todas essas diferentes respostas sob controle temporal e de magnitude de reforço em diferentes níveis. O fato de alguém relatar sentir fissura em uma situação pode ser mantido por um esquema, enquanto a autoadministração de determinado alimento (consumo alimentar propriamente dito) pode ser mantido por um esquema diferente, sequer concorrente com o anterior.

Os autores do presente estudo, portanto, não concordam com a inferência da literatura aqui apresentada de que indivíduos que relatem redução de fissura (suposta VD) também comeriam menos (suposta VD) após alguma instrução (suposta VI). Além das respostas de fissura e de comer se tratarem de duas respostas independentes (com variáveis controladoras possivelmente também diferentes, tendo a primeira um peso de componentes respondentes maiores que a segunda), o relato de fissura é ainda uma outra resposta a ser analisada, juntamente com suas variáveis controladoras.

Como discutido amplamente por Wechsler e Amaral (2009), a correspondência dizer-fazer (dizer "eu não comeria" e de fato não comer) e fazer-dizer (perceber sensação fisiológica que aprendeu culturalmente a nomear, em contexto semelhante, de fissura e dizer "eu senti muita fissura" 3 ) é antes de tudo, uma construção social que se dá, de forma independente, através das contingências de reforço típicas da comunidade verbal na qual a pessoa se insere, não devendo a primeira ser tida como uma promessa inquebrável e nem a segunda como um tato irrefutável (Skinner, 1957). A operacionalização dos termos utilizados e uma maior compreensão das diversas funções do comportamento verbal certamente reduziriam a probabilidade de cientistas em geral (e de neurocientistas, em particular) levantarem interpretações precipitadas, falsos problemas de pesquisa e/ou conceituarem de forma

3 Para discussão aprofundada acerca da nomeação de eventos privados, ver Tourinho (2009). pouco pragmática suas variáveis independentes e dependentes (para uma discussão mais aprofundada do quanto as neurociências se beneficiariam de um corpo filosófico-científico-conceitual mais estruturado, ver Bennet \& Hacker, 2003).

A presente pesquisa utilizou, como as pesquisas anteriores, autorrelatos de fissura para medir o quanto os participantes se descrevem como motivados a consumir os alimentos, porém usou o consumo desses alimentos como medida para evidenciar 1) se há correspondência entre o que os participantes dizem sentir perante os alimentos e o quanto eles consomem dos mesmos, 2) se as instruções exercem algum controle sobre o consumo alimentar dos participantes e 3) se as instruções exercem algum controle sobre os relatos de fissura.

Considerando as três perguntas (descritas acima) que esse experimento teve por objetivo responder, é possível afirmar que dentro das condições dispostas os dados obtidos e suas análises indicam que não há correspondência entre o relato de fissura e o consumo, já que os dados de fissura de cada participante não correspondem necessariamente com o quanto foi consumido de alimento. $\mathrm{O}$ fato de não ser possível afirmar que há correspondência entre fissura e consumo apresenta mais um empecilho à inferência feita pelos autores aqui citados, pois se não é possível constatar essa correspondência, torna-se impossível prever apenas por meio dos relatos de fissura qual seria o consumo de alimento dos participantes, justamente por não se poder tomar as correspondências verbais como fatos em si, existentes e reais, em vez de outra VD a ser analisada. Defende-se, portanto, que os relatos de fissura não podem ser considerados como única medida comportamental para evidenciar o quanto instruções controlam o comportamento alimentar.

Tendo em vista o consumo de alimentos, não é possível afirmar que há indicativos de que a instrução exerceu controle sobre o consumo dos alimentos, pois apesar da análise de média do consumo demonstrar um aumento para todos os participantes no decorrer das sessões, a análise de tendência no desenho de linha de base múltipla entre sujeitos apresenta indicativos divergentes. Por fim, considerando os dados de fissura e suas análises correspondentes, apesar dos relatos de fissura terem diminuído para os participantes 2 e 3 , considerando as análises das médias não é possível garantir que tal redução seja devida à inserção da VI, tendo-se em vista que para o Participante 3, com mais sessões de linha de base, a redução da fissura se deu ainda antes da inserção da VI.

Considerando os resultados apresentados tem-se indicativos de que as instruções não controlam os relatos de fissura e o consumo; e que os relatos de fissura não correspondem à quantidade de alimento consumida. Além disso, em conversa informal, duas participantes relataram terem se sentido incomodadas após as instruções terem sido apresentadas e disseram se lembrar de familiares que julgam a qualidade e quantidade dos alimentos que elas consomem. Dessa forma, é possível que o uso de instruções com o objetivo de redução de consumo de alimento não seja efetivo, possa produzir aversividade 
para indivíduos submetidos a intervenções como essa, produzindo inclusive alterações no consumo como forma de contracontrole a depender do histórico de cada participante.

É importante ressaltar que algumas variáveis não foram controladas suficientemente nesse experimento e podem ter influenciado o consumo das participantes. A qualidade dos alimentos é uma delas. É possível que o fato de alguns alimentos disponibilizados não serem industrializados os deixassem mais sujeitos a alterações de temperatura, qualidade, frescor e disponibilidade, o que pode ter influenciado o consumo dos participantes. Podese exemplificar o quanto a variação de um dado alimento pode influenciar o consumo, considerando a diferença de consumo entre a primeira e a segunda sessão de linha de base da Participante 2 (195g e 295 g, respectivamente). O número de participantes também pode ser uma limitação do presente trabalho, assim como o fato dos dados nas sessões de linha de base não demonstrarem estabilidade, já que houve limitação no número de sessões possíveis. Além disso, o fato de a coleta ter sido feita em dias diferentes pode ter afetado o controle do consumo, pois os participantes estavam a cada sessão expostos a variáveis ambientais diferentes (provas, prazos de entrega ou o quanto fizeram de atividades naquele dia).

Dessa forma, são necessárias novas pesquisas que controlem essas variáveis e que utilizem o delineamento de sujeito único para que seja possível coletar mais indicadores do quanto instruções podem, de fato, controlar a fissura e o consumo de alimentos altamente palatáveis.

\section{DECLARAÇÃO DE CONFLITO DE INTERESSES}

Todos os autores declaram que não há conflito de interesses de qual quer natureza relativos à publicação deste artigo.

\section{CONTRIBUIÇÃO DE CADA AUTOR}

Certificamos que todos os autores participaram suficientemente do trabalho para tornar pública sua responsabilidade pelo conteúdo. A contribuição de cada autor pode ser atribuída como se segue: N. M. Calegare e L. J. S. Dahás contribuíram para a concepção do artigo, sendo que a primeira autora realizou a coleta de dados inteiramente; N. M. Calegare, D. M. Regis Neto e L. J. S. Dahás foram responsáveis pela formulação do desenho metodológico; N. M. Calegare, D. M. Regis Neto e L. J. S. Dahás foram responsáveis pela redação final.

\section{DIREITOS AUTORAIS}

Este é um artigo aberto e pode ser reproduzido livremente, distribuído, transmitido ou modificado, por qualquer pessoa desde que usado sem fins comerciais. O trabalho é disponibilizado sob a licença Creative Commons 4.0 BY-NC.

\section{REFERÊNCIAS}

Albuquerque, L. C., \& Paracampo, C. C. P. (2010). Análise do controle por regras. Psicologia USP, 21, 253-273. doi: 10.1590/S0103-65642010000200004

American Psychiatric Association (2014). DSM-5: Manual diagnóstico e estatístico de transtornos mentais. Porto Alegre: Artmed.

Arcelus, J., Mitchell, A. J., Wales, J., \& Nielsen, S. (2011). Mortality rates in patients with anorexia nervosa and other eating disorders: a meta-analysis of 36 studies. Archives of General Psychiatry, 68, 724731. doi: 10.1001/archgenpsychiatry.2011.74

Baer, D. M., Wolf, M. M., \& Risley, T. R. (1968). Some current dimensions of Applied Behavior Analysis. Journal of Applied Behavior Analysis, 1, 91-97. doi: 10.1901/jaba.1968.1-91

Bennet, M. R., \& Hacker, P. M. S. (2003). Philosophical Foundations of Neuroscience. Malden: Blackwell Publishing.

Bighetti, F. (2003). Tradução e validação do Eating Attitudes Test (EAT-26) em adolescentes do sexo feminino na cidade de Ribeirão Preto - SP (Dissertação de Mestrado), Universidade de São Paulo, São Paulo.

Garner, D. M., \& Garfinkel, P. E. (1979). The Eating Attitudes Test: An index of the symptoms of anorexia nervosa. Psychological Medicine, 9, 273-279. doi: 10.1017/S0033291700030762

Hollmann, M., Hellrung, L., Pleger, B., Schlögl, H., Kabisch, S., Stumvoll, M., ... \& Horstmann, A. (2012). Neural correlates of the volitional regulation of the desire for food. International Journal of Obesity, 36, 648-655. doi: 10.1038/ijo.2011.125

Jansen, A. (2016). Eating disorders need more experimental psychopathology. Behaviour Research and Therapy, 86, 2-10. doi: 10.1016/j.brat.2016.08.004

Kazdin, A. E. (1982). Single case research designs. Methods for clinical and applied settings. New York, Estados Unidos: Oxford Universitu Press.

Kober, H., Mende-Siedlecki, P., Kross, E. F., Weber, J., Mischel, W., Hart, C. L., \& Ochsner, K. N. (2010). Prefrontal-striatal pathway underlies cognitive regulation of craving. Proceedings of the National Academy of Sciences, 107, 14811-14816.

McKim, W. A., \& Hancock, S. D. (2013). Drugs \& Behavior: An introduction to behavioral pharmacology. New Jersey: Pearson.

Ministério da Saúde, [MS], Secretaria de Vigilância em Saúde, Departamento de Vigilância de Doenças e Agravos não Transmissíveis e Promoção da Saúde (2015). Vigitel Brasil 2014: vigilância de fatores de risco e proteção para doenças crônicas por inquérito telefônico. Brasília, DF: Ministério da Saúde.

Pelchat, M. L., Johnson, A., Chan, R., Valdez, J., \& Ragland, D. (2004). Images of desire: food craving activation during fMRI. Neurolmage, 23, 1486-1493. doi: 10.1016/j.neuroimage.2004.08.023

Rachlin, H. (2000). The science of self-control. Cambridge: Harvard University Press. 
Raynor, H. A. \& Epstein, L. H. (2003). The relativereinforcing value of food under differing levels of food deprivation and restriction. Appetite, 40, 15-24. doi: 10.1016/S0195-6663(02)00161-7

Siep, N., Roefs, A., Roebroeck, A., Havermans, R., Bonte, M., \& Jansen, A. (2012). Fighting food temptations: the modulating effects of short-term cognitive reappraisal, suppression and up-regulation on mesocorticolimbic activity related to appetitive motivation. Neuroimage, 60, 213-220. doi: 10.1016/j.neuroimage.2011.12.067

Skinner, B. F. (1957). Verbal Behavior. New York: Appleton-Century-Crofts.

Skinner, B. F. (1969). An operant analysis of problem solving. Em B. F. Skinner (Org.), Contingencies of reinforcement: A theoretical analysis (pp. 133-171). New York, Estados Unidos: Appleton-Century-Crofts. (Trabalho original publicado em 1966)

Skinner, B. F. (1976). Causes and reasons. About behaviorism (pp. 132-150). New York, Estados Unidos: Random House. (Trabalho original publicado em 1974)

Tourinho, E. Z. (2009). Subjetividade e Relações Comportamentais. São Paulo: Paradigma.

Udo, T., \& Grilo, C. M. (2018). Prevalence and Correlates of DSM-5 Eating Disorders in Nationally Representative Sample of United States Adults. Biological Psychiatry, 84, 345-354. doi: 10.1016/ j.biopsych.2018.03.014

Wang, G. J., Volkow, N. D., Telang, F., Jayne, M., Ma, Y., Pradhan, K., ... \& Biegon, A. (2009). Evidence of gender differences in the ability to inhibit brain activation elicited by food stimulation. Proceedings of the National Academy of Sciences, 106, 1249-1254. doi: 10.1073/pnas.0807423106

Wechsler, A. M., \& Amaral, V. L. R., (2009). Correspondência Verbal: uma revisão da literatura. Revista Brasileira de Terapia Comportamental e Cognitiva, 11, 189-208.

Yokum, S., \& Stice, E. (2013). Cognitive regulation of food craving: effects of three cognitive reappraisal strategies on neural response to palatable foods. International Journal of Obesity, 37, 1565-1570. doi: 10.1038/ijo.2013.39

Zettle. R. D., \& Hayes, S. C. (1982). Rule governed behavior: A potential theoretical framework for cognitive therapy. In P. C. Kendall (Ed.), Advances in cognitive-behavioral research and therapy (pp. 73118). New York, NY: Academic.

Zvolensky, M. J, Lejuez, C. W., Stuart, G. L., \& Curtin, J. J. (2001). Experimental psychopathology in psychological science. Review of General Psychology, 5, 371-381. doi: 10.1037/1089-2680.5.4.371

Submetido em: 31/09/2018 Aceito em: 08/09/2019 